\title{
Comparison of Histological and Angiographic Measurements of the Basilar Artery in an Experimental Rabbit Subarachnoid Hemorrhage Model
}

\author{
Mert SAHINOGLU ${ }^{1}$, Oguz KARAKOYUN²${ }^{2}$, Fatih ALAGOZ³ , Deniz BILLUR ${ }^{4}$, Sevim AYDIN4, Rifat AKDAG \\ Ilkay AKMANGIT' ${ }^{6}$, Ergun DAGLIOGLU ${ }^{3}$ \\ ${ }^{1}$ Selcuk University, Faculty of Medicine, Department of Neurosurgery, Konya, Turkey \\ ${ }^{2}$ Ordu University Education and Research Hospital, Department of Neurosurgery, Ordu, Turkey \\ ${ }^{3}$ University of Health Sciences, Bilkent City Hospital, Department of Neurosurgery, Ankara, Turkey \\ ${ }^{4}$ Ankara University, Faculty of Medicine, Department of Histology and Embryology, Ankara, Turkey \\ ${ }^{5}$ Sevket Yilmaz Education and Research Hospital, Department of Neurosurgery, Bursa, Turkey \\ ${ }^{6}$ University of Health Sciences, Bilkent City Hospital, Department of Radiology, Ankara, Turkey \\ Corresponding author: Ergun DAGLIOGLU edaglioglu@gmail.com
}

\section{ABSTRACT}

AIM: To compare the histological and angiographic measurements of the basilar artery in an experimental rabbit subarachnoid hemorrhage model.

MATERIAL and METHODS: The basilar artery was measured using both histological and angiographic methods in experimental subarachnoid hemorrhage (SAH) and vasospasm rabbit models. New Zealand white rabbits were randomly categorized into two groups: control and SAH groups. The SAH group rabbits were operated on to create an experimental SAH. Both groups were examined angiographic and histological methods.

RESULTS: On comparing the two methods, angiographic and histopathological measurements of the basilar artery were similar in the control group. However, in the SAH group, the difference between the angiographic and histopathological measurement methods was significant. Histopathological measurements of the basilar artery were lower than angiographic measurements, and the difference was statistically significant. In the angiographic method, although there was a marked decrease in basilar artery measurements in the SAH group, the differences between the groups was not statistically significant. However, in the histopathological method, measurement differences between the control and SAH groups were statistically significant.

CONCLUSION: Histopathological measurements were shown to be more sensitive than angiographic methods in demonstrating cerebral vasopasm in experimental SAH rabbit models.

KEYWORDS: Angiography, Basilar artery, SAH model

ABBREVIATIONS: SAH: Subarachnoid hemorrhage, DSA: Digital subtraction angiography, HCl: Hydrochloride, CSF: Cerebrospinal Fluid, TI: Tunica intima, TM: Tunica media, TA: Tunica adventitia, H\&E: Hematoxylin and eosin, SPSS: Statistical Package for the Social Sciences

Mert SAHINOGLU (1) : 0000-0003-0633-8304 Oguz KARAKOYUN (1) : 0000-0002-1306-7584 Fatih ALAGOZ (1) : 0000-0001-6910-0585
Deniz BILLUR (1) : 0000-0001-8541-8251

Sevim AYDIN (D): 0000-0003-2445-2346

Rifat AKDAG (1) : 0000-0001-7638-8361
Ilkay AKMANGIT (1) : 0000-0002-6553-3639 Ergun DAGLIOGLU (1) : 0000-0002-7162-3999 


\section{INTRODUCTION}

A neurysmal rupture is the major cause of spontaneous subarachnoid hemorrhage $(\mathrm{SAH})$, and it has a high mortality rate, both before and after admission to the hospital (6). Up to $30 \%$ of survivors have significant morbidity, leading to decreased quality of life in most patients (18). In a long-term study of aneurysmal SAH survivors for $>20$ years, only $40 \%$ of patients reported a return to their normal lifestyles (16). With recent advances in diagnosis and treatment of aneurysms, cerebral vasospasm is now considered the most important complication for morbidity and mortality. Cerebral vasospasm is a specific type of vascular contraction leading to decreased perfusion at the distal areas of the affected major cerebral arteries. It can be irreversible or slightly reversible and result in cerebral ischemia $(4,9)$. It usually occurs on day 3 after $\mathrm{SAH}$, peaks on days 6 and 8 , and lasts for 2-3 weeks (20). Ongoing studies to understand its mechanism have led to many theories, and it is considered to be multifactorial.

Factors such as microcirculatory dysfunction, microthrombosis, and cortical spreading depression as well as early brain injury, inflammation, and oxidative stress play a significant role in pathogenesis $(2,3)$. Intrinsic factors that cause vasoconstriction, mechanical factors, apoptosis, immunoreactivity, or inflammatory events are investigated in the pathogenic process, but effective treatments are still limited and many patients either die due to secondary ischemia or continue living with severe neurological deficits. In some patients, SAH may be radiologically observed before symptoms develop. Symptomatic vasospasm is observed in $20 \%-30 \%$ of patients but can be documented in $30 \%-70 \%$ of patients by angiography 1 week after SAH development $(7,11)$. This provides a good advantage for physicians to plan the treatment and follow-up. However, it is not clear when neurological deficits occur in patients with radiological vasospasm, and a classification regarding this subject is lacking. Currently, digital subtraction angiography (DSA) is the gold standard method and most effective radiological tool for the diagnosis of spontaneous $\mathrm{SAH}$. However, the degree of narrowing of the cerebral artery diameter that leads to neurological deficits is unknown.

Measurements using DSA are limited to the inner arterial diameter of the cerebral vasculature. However, the arterial diameter of the outer layer, lumen area, and vessel wall thickness can possibly be measured histologically. Previous studies clearly demonstrated that the lumen diameter and vessel wall area and thickness were correlated with the degree of vasospasm $(5,13,17)$. In this study, basilar artery measurements were performed using both histopathological and angiographic methods in an experimental rabbit $\mathrm{SAH}$ model, and the results were compared to determine the correlation between the two methods.

\section{MATERIAL and METHODS}

This study was approved by the Animal Studies Local Ethics Committee of the Ministry of Health, Ankara Training and Research Hospital (date: October 1, 2013; Decree No: 164), and conducted at the experimental animal laboratory in the same hospital. A total of 16 New Zealand white rabbits weighing 2,140-3,210 g were used in the study. They were housed in standard conditions and provided with sufficient food and water throughout the experimental course. The rabbits were randomly distributed into two groups: control group, in which no surgical procedures were performed, and $\mathrm{SAH}$ group, in which an experimental SAH was created using the surgical technique described below.

\section{Surgical Procedures}

After a 6-hour fasting period, ketamine hydrochloride $50 \mathrm{mg} /$ kg (Ketaset ${ }^{\circledR}$ Ketamine HCl Injection, USP, Fort Dodge ${ }^{\circledR}$, lowa 50501 USA) and xylazine $10 \mathrm{mg} / \mathrm{kg}$ (Rompun ${ }^{\circledR} 2 \%$, Bayer, Leverkusen, Germany) were intramuscularly administered. The rabbits were allowed to breathe spontaneously thereafter. While the rabbits were in the prone position, a $3-\mathrm{cm}$ vertical skin incision was made at the occipital bone and $\mathrm{C} 1$ vertebral regions. Following the dissection of the occipitocervical muscles from the medial to lateral direction and craniovertebral junction exposure, the dura was observed between the occipital bone and C1. Craniectomy was performed at the suboccipital bone using microforceps, followed by a cisterna magna puncture using a 1-ml injector with a 23-G needle. Following the drainage of approximately $0.8-1-\mathrm{ml}$ cerebrospinal fluid, the injector needle tip was fixed. The same amount $(0.8-1 \mathrm{ml})$ of blood taken from the central ear artery of the rabbit was injected into the cisterna magna using the fixed needle tip to generate the experimental $\mathrm{SAH}$. The rabbits were maintained under standard conditions for 72 hours postoperatively.

\section{Cerebral Angiographic Imaging}

Cerebral angiography was performed 72 hours after blood injection to each rabbit's cisterna. After anesthesia following the experimental protocol, the control and $\mathrm{SAH}$ group rabbits were stabilized in the supine position on an angiography table (General Electric Medical Systems, cerebral angiography device, 2008, Milwaukee, USA). The aim of angiography was to radiologically show if the basilar artery exhibited vasospasm after SAH. The right femoral artery was used in most rabbits by introducing an introducer wire of a Balt 3-F introducer set through a blue intravenous line (22G) and placing the Balt 3-F introducer sheath over the wire into the artery. The main carotid artery or vertebral artery to be visualized was catheterized with a Cordis Prowler 21 select plus microcatheter using a Stryker Transcend hydrophilic 0.0014 micro guidewire EX tip. Next, angiographic runs were acquired using Ultravist 300 (Schering, Germany) contrast material including $300 \mathrm{mg} / \mathrm{mL}$ iodine, diluted 1:2 for microcatheter injection. The raw image was reconstructed using Osirix World's fastest Dicom Viewer version 5.0.2 software. Digital measurements of the arteries were performed after bone substraction and calibration with the catheter diameter. Arterial diameters were measured at three different points of the basilar artery, and the mean diameter of the basilar artery was calculated from these three measurements (Figure 1).

\section{Preparation of the Cerebral Tissues}

To ensure that there was no significant difference between 


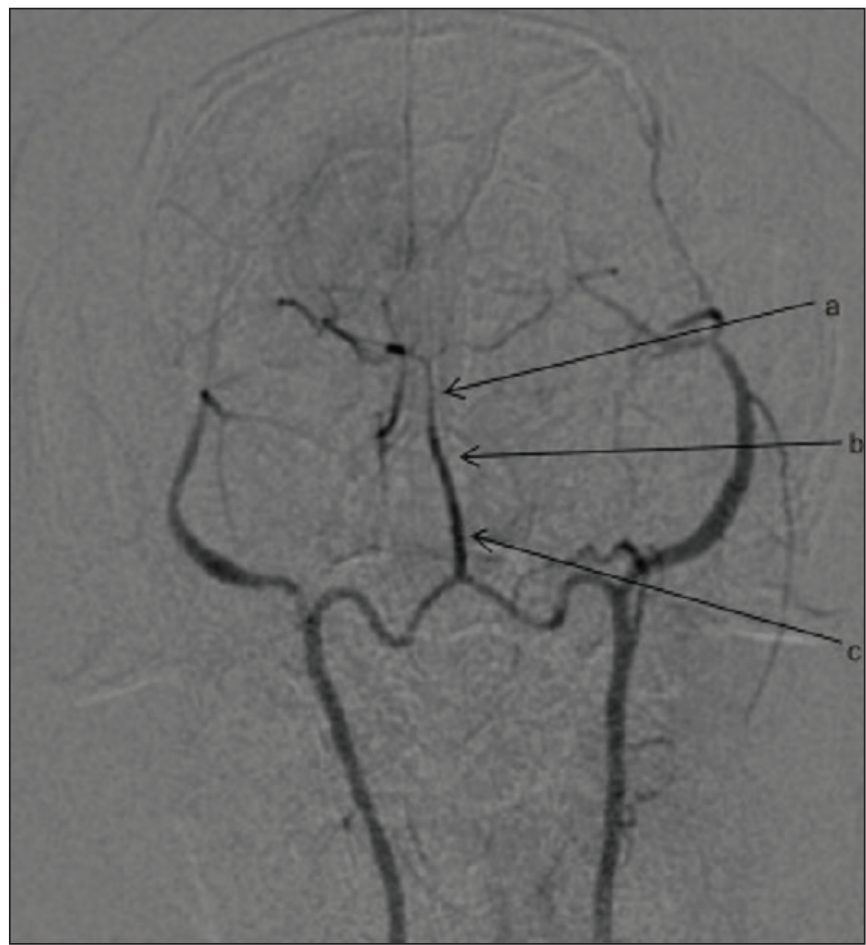

Figure 1: The diameter of the basilar artery was measured in three different segments. a: distal $1 / 3^{\text {rd }}$; b: middle $1 / 3^{\text {rd }}$; and c: proximal $1 / 3^{\text {rd }}$.

the histopathological and angiographic measurements, the rabbits were sacrificed $\leq 6$ hours after angiography. After the induction of general anesthesia and thoracotomy, the descending aorta was clamped, an 8-F nelaton catheter was placed into the left ventricle, and approximately $1,000 \mathrm{~mL}$ of Ringer's lactate solution was injected. An incision was made in the right atrium to drain the blood returning to the right heart following brain perfusion. After brain perfusion, craniectomy was performed, sparing the suboccipital fossa and preserving the cerebral hemispheres, cerebellum, brain stem, and upper cervical cord. Microsurgical techniques were carefully used in an attempt to preserve the basilar artery and remove the brain stem and cisternae in their physiological localization as a single piece. No microsurgical instrument was brought into contact with the basilar artery during the procedure. The excised cerebral tissues were preserved in 10\% formaldehyde solution at $+4{ }^{\circ} \mathrm{C}$ and used for light microscopic examination.

\section{Light Microscopic Examination}

Tissues from both groups were examined under a surgical microscope to ensure that the basilar artery was in its bed and, for the SAH group, the presence of surrounding blood in the subarachnoid space. For the histological examination, the basilar artery was dissected and divided into three pieces: 1/3 upper, mid, and lower sections, together with the neighboring brain stem tissue. The tissues were fixed in buffered $10 \%$ formalin solution at least $72 \mathrm{~h}$ for approximately 7 days. Then, routine protocols for histological examination were performed for washing, dehydration, and embedding in paraffin blocks. Tissue sections of $5-\mu \mathrm{m}$ thickness were prepared using a Leica
Table I: Number of Rabbits and the Mortality of the Study

\begin{tabular}{lccc}
\hline & $\begin{array}{c}\text { Number of } \\
\text { total rabbit }\end{array}$ & $\begin{array}{c}\text { Control } \\
\text { group }\end{array}$ & SAH group \\
\hline Before study & 16 & 8 & 8 \\
\hline End of the study & 16 & 8 & 8 \\
\hline Mortality & 0 & 0 & 0 \\
\hline
\end{tabular}

RM 2125RT sliding microtome, stained with hematoxylin and eosin, and examined under $20 \times$ and $40 \times$ magnifications using a light microscope (Carl Zeiss Axio Scope-A1, Germany). The inner diameters of the arterial sections at three different levels were measured from the endothelial to endothelial surface in five different quadrants using a light microscope and Axiovision software program. The arithmetic mean of the diameter of the basilar artery was calculated from these three measurements.

\section{Statistical Analysis}

Statistical analysis was performed using SPSS version 18.0 software package (An IBM Company, Westlands Road, Quarry Bay, Hong Kong). Descriptive analyses were performed using data of the SAH and control groups. Comparative analysis between groups was performed using the Mann-Whitney $U$-test and Wilcoxon's paired test.

\section{RESULTS}

Overall, 16 rabbits were included in the study. No rabbit died during the study (mortality rate, 0\%) (Table I).

\section{Light Microscopy Findings}

In the control group, the basilar artery had a normal appearance under light microscopy. The tunica intima (TI) and endothelial cells also showed normal appearance. The elastic lamina was thin and unfolded. The smooth muscle cells were concentrically oriented in the tunica media (TM) (Figure 2A). In the SAH group, the lumen of the basilar artery was narrowed, and its wall thickness was significantly increased. Endothelial cells were irregular in appearance, and the internal elastic membrane was markedly folded (Figure 2B).

The basilar artery lumen diameter was measured in histological sections and compared between the groups. The basilar artery lumen diameter measurements in the SAH group were lower than those in the control group, except for two rabbits, indicating that the basilar artery lumen diameter was narrowed in the SAH group (Figure 3A, B). When histological measurements were compared, there was a significant difference $(p<0.05)$ in the mean distribution between the control and SAH group, with 95\% confidence level (CL). The mean diameters were $0.41 \mathrm{~mm}$ and $0.22 \mathrm{~mm}$ in the control and $\mathrm{SAH}$ groups, respectively $(\mathrm{p}=0.015)$.

\section{Cerebral Angiography Findings}

Cerebral angiography images were used to measure the basilar artery lumen diameters in both groups (Figure 4A, $\mathrm{B})$. In the SAH group, the proximal, middle, and distal $1 / 3$ 

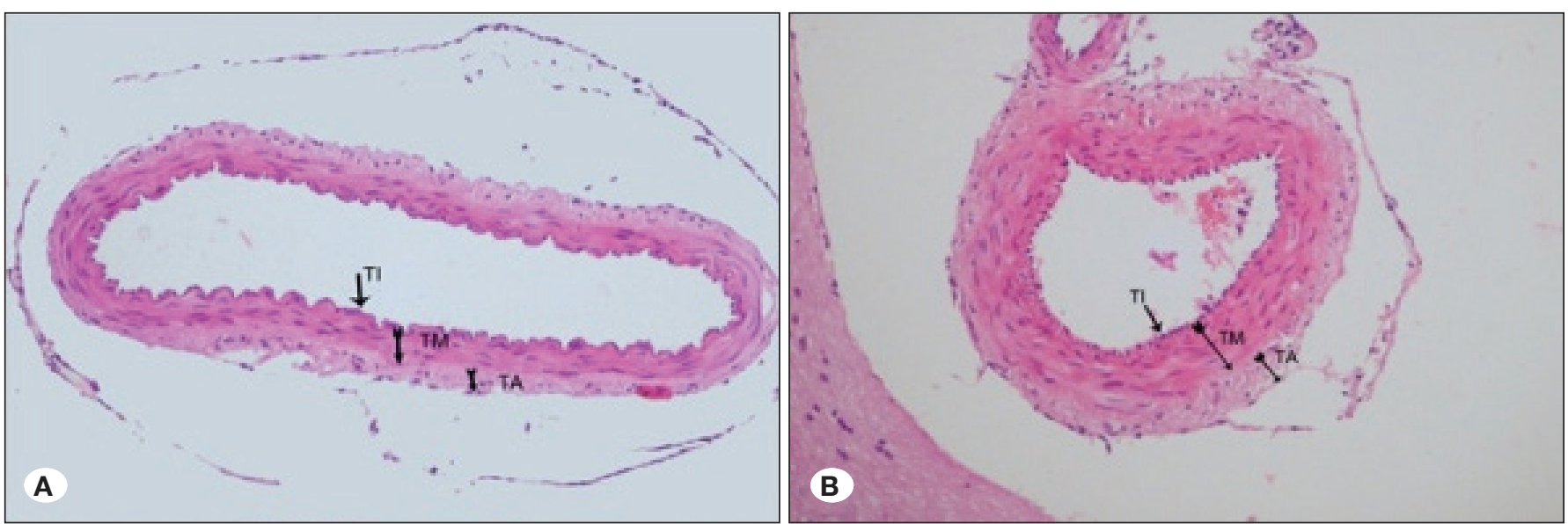

Figure 2: Light microscopy findings (H\&E staining, 200x). A) Normal tunica intima (TI), tunica media (TM), and tunica adventitia (TA) layers in the control group; B) luminal narrowing and markedly increased wall thickness in the SAH group.
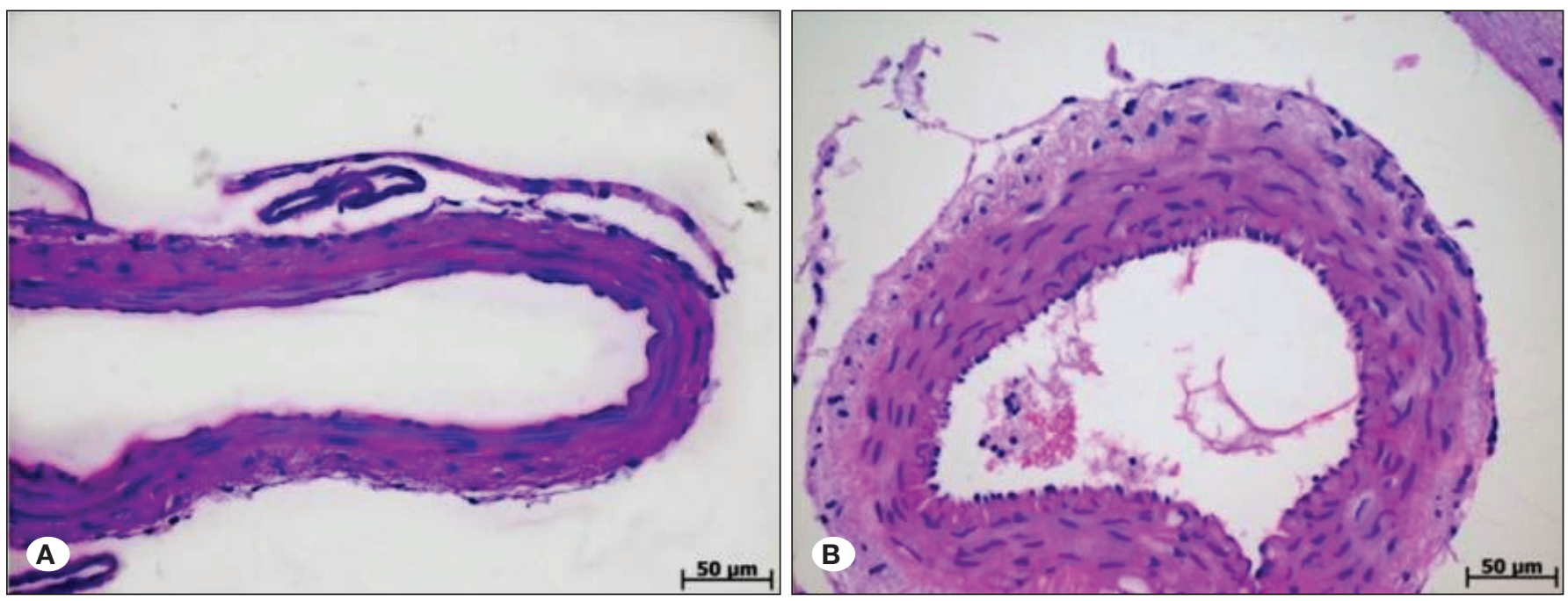

Figure 3: H\&E staining, 40×. A) Basilar artery in the control group,

B) basilar artery in the SAH group.

sections of the basilar artery were measured after calibration with the catheter diameter. The arithmetic mean of these three measurements was used for comparison (Table II).

When angiographic measurements of the basilar artery diameters were compared, the SAH group displayed narrowing of the lumen diameter. However, the Mann-Whitney U-test revealed no significant difference with $95 \% \mathrm{Cl}(\mathrm{p}>0.05)$ between the mean diameters in the two groups. The mean diameter was $0.45 \mathrm{~mm}$ in the control group and $0.36 \mathrm{~mm}$ in the $\mathrm{SAH}$ group $(p=0.156)$.

In the control group, the diameters of the basilar artery lumens were narrower when measured using histology than when measured using angiography. However, the difference between the two measurements was not statistically significant $(p>0.05)$ with $95 \% \mathrm{Cl}$. The angiographic mean was $0.45 \mathrm{~mm}$ and the histological mean was $0.41 \mathrm{~mm}$ the control group $(p=0.40)$. In the SAH group, the diameters of the basilar artery lumens were narrower when measured using histology than when measured using angiography (Table III). A
Table II: Comparison Between Histopathological and Angiographic Measurements $(\mathrm{mm})$ of Basilar Artery Lumen Diameters in the $\mathrm{SAH}$ Group

\begin{tabular}{ccc}
\hline $\begin{array}{c}\text { Rabbit } \\
\text { number }\end{array}$ & $\begin{array}{c}\text { Histological } \\
\text { measurement }\end{array}$ & $\begin{array}{c}\text { Angiographic } \\
\text { measurement }\end{array} \quad \mathbf{p}$ \\
\hline $\mathbf{1}$ & 0.21847 & 0.28 \\
\hline $\mathbf{2}$ & 0.20256 & 0.43 \\
\hline $\mathbf{3}$ & 0.20236 & 0.41 \\
\hline $\mathbf{4}$ & 0.18549 & 0.59 \\
\hline $\mathbf{5}$ & 0.18006 & 0.42 \\
\hline $\mathbf{6}$ & 0.22093 & 0.29 \\
\hline $\mathbf{7}$ & 0.12415 & 0.12 \\
\hline $\mathbf{8}$ & 0.39955 & 0.375 \\
\hline
\end{tabular}


Table III: Mean Measurements $(\mathrm{mm})$ and Analysis Results Conducted Using the In-Group Mann-Whitney U-test and Wilcoxon Paired Test of the Control and SAH Groups

\begin{tabular}{lcc}
\hline & Control group & SAH group \\
\hline Histological & 0.41 & 0.22 \\
\hline Angiographic & 0.45 & 0.36 \\
\hline $\mathbf{p}$ & 0.40 & $\mathbf{0 . 0 2 4}$ \\
\hline
\end{tabular}

statistically significant difference was observed between the mean measurements $(p<0.05)$ with $95 \% \mathrm{Cl}$. The angiographic mean was $0.3 \mathrm{~mm}$ and the histological mean was $0.22 \mathrm{~mm}$ in the SAH group $(p=0.024)$.

\section{DISCUSSION}

In this study, histological and angiographic methods of basilar artery measurements were compared in experimentally generated SAH and vasospasm rabbit models. SAH development and vasospasm after 72 hours were confirmed by cerebral angiography measurements. After sacrificing the rabbits, the basilar artery wall thickness was histologically measured, and measurements were statistically compared.

In experimental SAH models, the vasospasm should be decreased by histomorphometric or angiographic measurements in order to evaluate the treatment effect. The arterial diameter can be shown via intra-arterial microcatheter calibration measurements for standardized selective cerebral digital angiography measurement. Weidauer et al. showed that standardized angiography measurements can be performed even in experimental SAH rat models (19). However, using small animals may be problematic for studies of cerebral vasospasm because their arterial diameters are small. Therefore, the sensitivity of cerebral angiography measurements is weaker in studies with rats and mice $(1,8)$; however, the sensitivity can be precisely measured in rabbits and dogs $(14,15)$.

Using DSA, the diameter of the cerebral artery is measured between two opposite internal surfaces of the arterial wall. In experimental SAH and vasospasm model studies, the section where basilar artery measurements are performed should be identified. In the literature, the basilar artery middle $1 / 3$ section is mostly preferred. However, in cerebrovascular structures where anatomical variations are frequent, the error measurement rates are higher; thus, multiple measurements are needed. While Longo et al. took the basilar artery middle 1/3 section as the basis for angiographic measurement, Koktekir et al. emphasizes that the mean of three different segments of the middle point of the basilar artery should be measured to ensure anatomical standardization $(8,10)$. In our study, the mean of three measurements were used to avoid these errors in both histological and angiographic measurements, and statistical means were used for comparisons.

In DSA, the internal lumen of the artery in which the contrast material passes through corresponds to the measurement from the intima to the opposite arterial intima in the histopathological method. In histological analysis, the outer diameter of the arterial wall, lumen area, and wall thickness can be measured. However, histological measurements are performed after animal sacrifice and tissue processing and are not performed simultaneously. Even if appropriate methods are used, technical procedures can cause variations that affect the measurement accuracy. Moreover, a disadvantage of histological measurements is that they cannot be repeated, whereas angiographic measurements can be performed repeatedly (8).

In experimental SAH models, the delicate balance between vasodilatation and vasoconstriction at the cerebral endothelial surface is disrupted due to biochemical changes. Pathomorphological changes such as endothelial folding or desquamation and vacuolization and fibrous tissue formation between the endothelial and muscular layers can be observed (14). In light microscopic studies on cerebral vasospasm in rabbit SAH models, increased basilar artery wall thickness, marked endothelial disruption, and increased folding of the internal elastic membrane were observed. However, damage to the basilar artery during decapitation and postmortem dissections can affect the results of the study (12). Tissue preparation procedures for histopathological analysis may also lead to false-positive or false-negative findings of the vasospasm. Careful dissections and multiple measurements can be performed to minimize sampling errors. In the present study, although these errors were observed in two rabbits, the statistical analysis showed a statistically significant difference in histological measurements between the control and $\mathrm{SAH}$ groups, with the $p$-value of 0.015 .

Our angiographic measurements showed that basilar artery lumen diameters of rabbits in the SAH group were narrower than those of rabbits in the control group. However, the MannWhitney U-test revealed no significant difference between the two groups $(p=0.156)$. This finding can be attributed to the difficulty of angiographic measurement despite accurate calibrations and the effect of using small experimental animals. Rabbits were used in the present study to eliminate this negative effect; however, it is important to perform angiography in animals. Another advantageous factor for the use of rabbits is the lower experimental costs than that of other animals. Furthermore, surgery is easier to perform because their anatomy is larger than those of mice and rats.

When comparing angiographic and histological measurements in the SAH group, histological measurements showed significantly narrower lumen diameters, demonstrating that histological measurements are more effective in diagnosing cerebral vasospasm. The fact that all histological measurements were lower than angiographic measurements is an important finding of this study. In the SAH group, remarkable thickening of the vessel wall, irregular appearance of endothelial cells, and increased folds from the elastica interna to the membrane were noteworthy. In the control group, light microscopic examination of the basilar artery revealed that the vessels had a normal appearance. In the TI layer, the endothelial cells appeared to be normal, and the elastic laminate was thin and not curled. In the TM layer, smooth muscle cells 


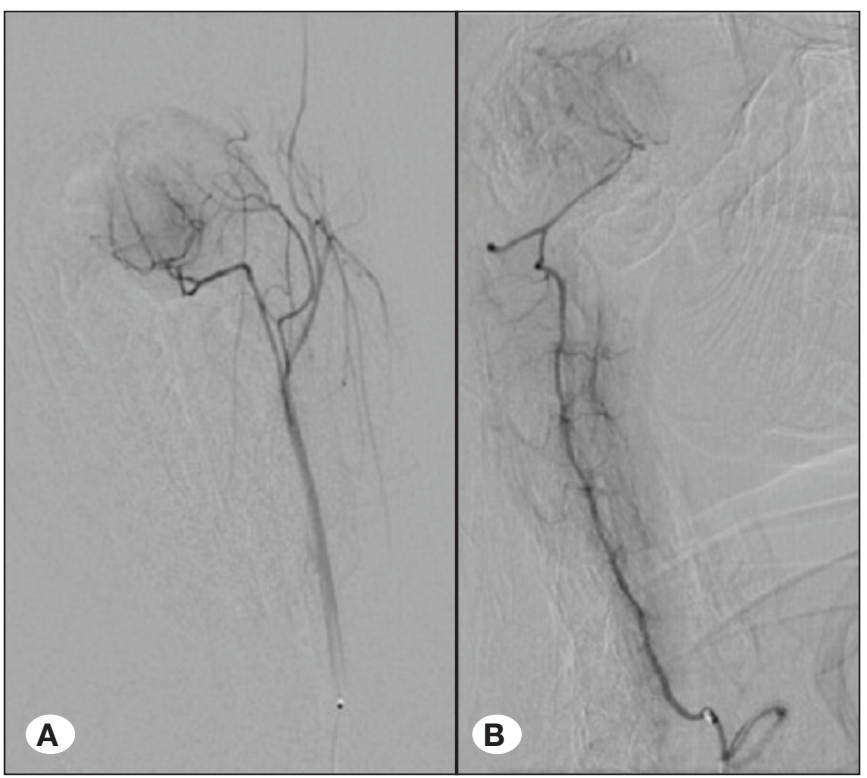

Figure 4: A) Angiography image in the control group; B) lateral angiographic image in the SAH group: basilar artery and branches after the left vertebral artery injection.

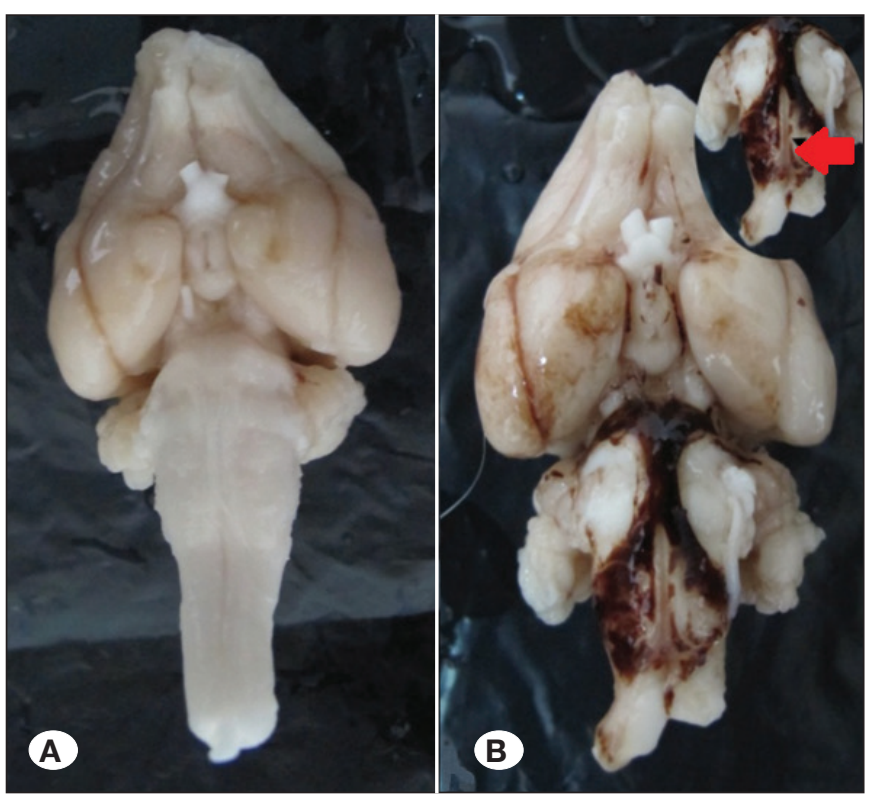

Figure 5: A) Basal view of the cerebellum and brainstem, which was removed in one piece of the rabbit, in the control group. B) Bottom view of the cerebellum and brainstem, which was removed in one piece, in the SAH group (the basilar artery is marked with red arrow). Clotted blood is seen in the cistern and basilar artery.

were observed to be in a concentric arrangement. Thus, lower basilar artery lumen measurements were observed in the SAH group.

This study has some limitations. This study model was established by injecting blood into the cisterna magna with a single injection. However, this can also be performed with a double injection. Although the double-injection procedure has a risk of increasing mortality in experimental animals, it has an advantage in terms of establishing a model closer to $\mathrm{SAH}$ in humans. Single-injection modeling was previously used because of the lack of experimental animals in the study (17). There was no cases of rabbit mortality during the study. Even in rat studies, the mortality rate varies between $1.5 \%$ and $47 \%$ (5). Although a certain amount of blood was injected using cisternal injection in rabbits, radiological imaging and $\mathrm{SAH}$ grading, which accurately show the degree of SAH generated in each rabbit, could not be performed due to the physical conditions. Because the center where the experiment was performed and the center where radiological imaging was performed were far apart, radiological imaging and $\mathrm{SAH}$ grading were not performed to prevent the rabbits from experiencing stress during transport and not increasing the mortality rate. However, there was sufficient macroscopic and microscopic evidence that there was sufficient blood around the cisterna and the basilar artery at the time of sacrifice (Figure $5 \mathrm{~A}, \mathrm{~B})$. Another limitation was that there was a time difference of approximately $6 \mathrm{~h}$ between angiography and preparation of histopathological sections. This was caused by the distance between the angiography center and the laboratory where rabbits were sacrificed. In addition, hyperselective vertebral artery catheterizations should be avoided during cerebral angiographic imaging of rabbits. Findings of increased vasospasm after catheterization with a microguidewire can be monitored and may be an obstacle to accurate measurement. Therefore, proximal injections should be preferred as much as possible for angiographic measurements.

\section{CONCLUSION}

This study compared histopathological and angiographic methods of measurements of vasospasms occurring after experimental $\mathrm{SAH}$ and found that histological measurements yield more reliable results. This may be due to difficulties of standardization of in vivo angiographic measurements in rabbits.

\section{ACKNOWLEDGEMENTS}

The preparation for publication of this article is partially supported by the Turkish Neurosurgical Society.

\section{REFERENCES}

1. Altay T, Smithason S, Volokh N, Rasmussen PA, Ransohoff RM, Provencio JJ: A novel method for subarachnoid hemorrhage to induce vasospasm in mice. J Neurosci Methods 183(2): 136-140, 2009

2. Brathwaite S, Macdonald RL: Current management of delayed cerebral ischemia: Update from results of recent clinical trials. TransI Stroke Res 5(2):207-226, 2014

3. Ciurea AV, Palade C, Voinescu D, Nica DA: Subarachnoid hemorrhage and cerebral vasospasm - Literature review. J Med Life 6(2):120-125, 2013

4. Dorsch NW: Cerebral arterial spasm-a clinical review. $\mathrm{Br} \mathrm{J}$ Neurosurg 9(3):403-412, 1995 
5. Dudhani RV, Kyle M, Dedeo C, Riordan M, Deshaies EM. A low mortality rat model to assess delayed cerebral vasospasm after experimental subarachnoid hemorrhage. J Vis Exp 71: 4157, 2013

6. Hijdra A, Braakman R, van Gijn J, Vermeulen M, van Crevel $\mathrm{H}$ : Aneurysmal subarachnoid hemorrhage. Complications and outcome in a hospital population. Stroke 18(6):1061-1067, 1987

7. Kassel NF, Sasaki T, Colohan ART, Nazar G: Cerebral vasospasm following aneurysmal subarachnoid hemorrhage. Stroke 16:562-572, 1985

8. Koktekir E, Erdem Y, Bayar MA, Gokcek C, Karatay M, Kilic C: A new approach to the treatment of cerebral vasospasm: The angiographic effects of tadalafil on experimental vasospasm. Acta Neurochir 152:463-469, 2010

9. Kolias AG, Sen J, Belli A: Pathogenesis of cerebral vasospazm following aneurysmal subarachnoid hemorrhage: Putative mechanisms and novel approaches. J Neurosci Res 87:1-11, 2009

10. Longo M, Blandino A, Ascenti G, Ricciardi GK, Granata S, Vinci S: Cerebral angiography in the rat with mammographic equipment: A simple, cost-effective method for assessing vasospasm in experimental subarachnoid haemorrhage. Neuroradiology 44:689-694, 2002

11. Mayberg MR, Batjer HH, Dacey RG Jr, Diringer M, Haley EC Jr, Heros RC, Sternau LL, Torner J, Adams HP Jr, Feinberg W, Thies W: Guidelines for the management of aneurysmal subarachnoid hemorrhage: A statement of healthcare professionals from aspecial writing group of Stroke Council, American Heart Association. Circulation 90:2592-2605, 1990

12. Megyesi JF, Volrath B, Cook DA, Findlay JM: In vivo animal models of cerebral vasospasm: A review. Neurosurgery 46(2): 448-461, 2000
13. Raslan F, Albert-Weißenberger C, Westermaier T, Saker S, Kleinschnitz C, Lee JY: A modified double injection model of cisterna magna for the study of delayed cerebral vasospasm following subarachnoid hemorrhage in rats. Exp Translational Stroke Med 4:23, 2012

14. Seifert V, Stolke D, Reale E: Ultrastructural changes of the basilar artery following experimental subarachnoid haemorrhage. A morphological study on the pathogenesis of delayed cerebral vasospasm. Acta Neurochir (Wien) 100(3-4): 164-171, 1989

15. Solmaz I, Onal MB, Civelek E, Kircelli A, Ongoru O, Ugurel S, Erdogan E, Gonul E: The effects of intrathecal nicergoline and nimodipine in cerebral vasospasm: An experimental study in rabbits. Acta Neurochir Suppl 110(Pt 2):81-85, 2011

16. Sonesson B, Kronvall E, Säveland H, Brandt L, Nilsson OG: Long-term reintegration and quality of life in patients with subarachnoid hemorrhage and a good neurological outcome: findings after more than 20 years. J Neurosurg 128(3):785792, 2018

17. Titova E, Ostrowski RP, Zhang JH, Tang J: Experimental models of subarachnoid hemorrhage for studies of cerebral vasospasm. Neurol Res 31:568-581, 2009

18. Wardlaw JM, White PM: The detection and management of unruptured intracranial aneurysms. Brain 123(Pt2):205-221, 2000

19. Weidauer S, Vatter H, Dettmann E, Seifert V, Zanella FE: Assessment of vasospasm in experimental subarachnoid hemorrhage in rats by selective biplane digital subtraction angiography. Neuroradiology 48:176-181, 2006

20. Wilkins RH: Cerebral vasospasm. Crit Rev Neurobiol 6(1):5177,1990 